EDITORIAL OPEN

\title{
Standards for reporting implementation studies (StaRI): enhancing reporting to improve care
}

npj Primary Care Respiratory Medicine (2017)27:42 ;

doi:10.1038/s41533-017-0045-7

The npj Primary Care Respiratory Medicine is extending its scope to include implementation science. Service delivery and organisation of healthcare has always been part of the journal's remit, and many of the published papers already reflect the challenges of implementing evidence-based care, ${ }^{1-11}$ so this change is making explicit a long-held interest of the journal, its authors and readers. The International Primary Care Respiratory Group (IPCRG) in its Research Needs Statement prioritised real-life studies evaluating 'overall management strategies' over efficacy trials of specific drugs or treatments. ${ }^{12,13}$ Similarly, the Primary Care Respiratory Society (PCRS-UK) includes 'promoting and disseminating real life primary care research in respiratory conditions to support policy and education activities' as one of its core activities. ${ }^{14}$ This extension of emphasis therefore resonates with both the parent primary care organisations.

Implementation science is a relatively new discipline that aims to develop the evidence base on how to translate research findings into routine care. ${ }^{15,16}$ This is a broad agenda which extends from preliminary work exploring the factors affecting implementation, 30, 11 developing implementation strategies, ${ }_{1}^{1}$ piloting the processes, ${ }^{2}$ testing the impact of implementation, ${ }^{4,8,9}$ and promoting scaling up and sustainability. ${ }^{6}$ This has been conceptua- lised as an additional cycle which takes an effective complex intervention, ${ }^{17}$ explores the existing context, and develops and evaluates strategies for embedding change in routine care. ${ }^{18}$ Other models have extended the spectrum of efficacy and effectiveness trials to encompass implementation science (see Fig. 1). ${ }^{19}$ The underlying tenet is the same: interventions may be effective in trials, but clinicians and healthcare organisations struggle to introduce them in the busy complex world of routine clinical practice. This may be a particular challenge in the context of primary care where a multitude of guidelines may be applicable to the diverse demands of front-line medical practice. A classic example is the poor implementation of self-management for asthma despite a long-standing and overwhelmingly positive evidence base. ${ }^{20,21}$ Understanding the routines of primary care practice is the first step in developing a workable strategy that enables self-management to be embedded in practice. ${ }^{11}$

Existing methodologically-based reporting standards (e.g., CONSORT, ${ }^{22}$ STROBE, $^{23}$ COREQ $^{24}$ ) are not wholly appropriate for reporting implementation studies, though some of the methodological criteria will be useful. It is for this reason that we, together with colleagues, launched the Standards for Reporting Implementation studies (StaRI) initiative. npj PCRM will be encouraging authors of implementation studies to use StaRI, recently published in the $\mathrm{BMJ}^{25}$ with an accompanying explanation and elaboration document. $^{26}$ The checklist for authors to complete is freely available from http://www.bmj.com/content/bmj/suppl/2017/03/06/bmj.

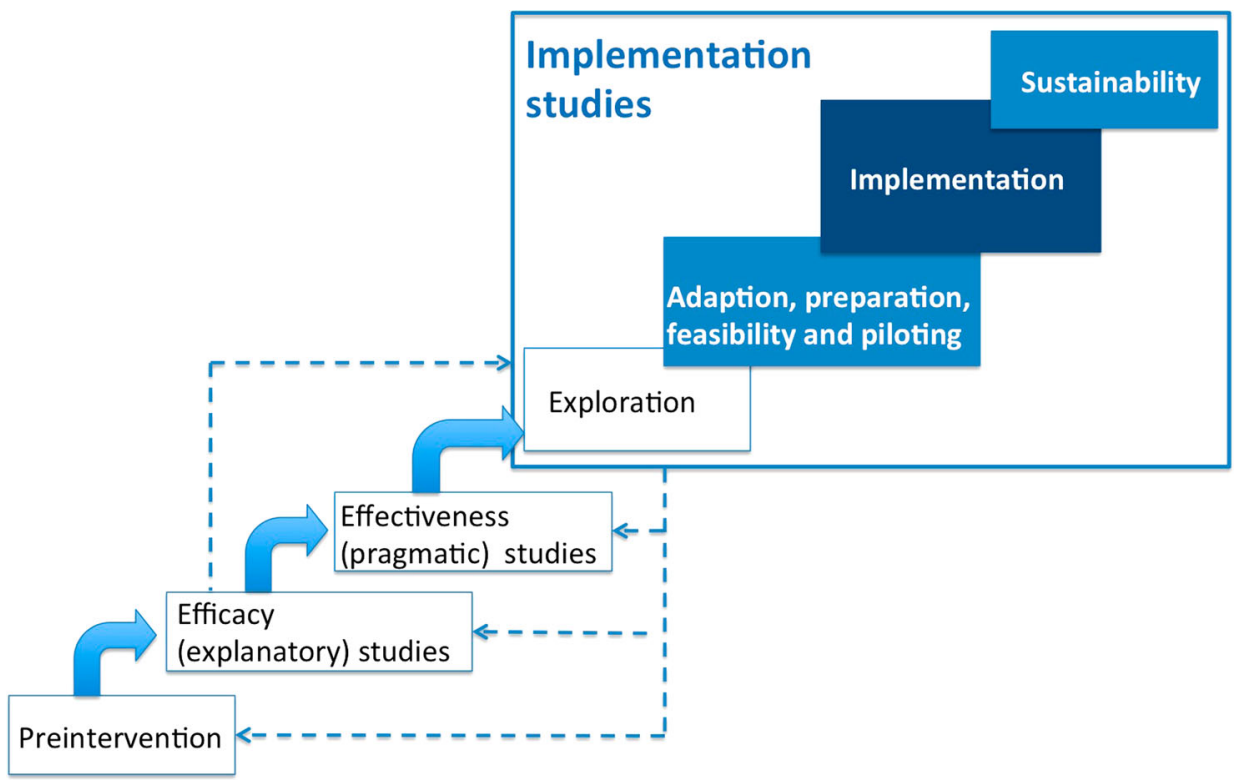

Fig. 1 Positioning of implementation studies and the focus of StaRI reporting standards (reproduced with permission from Pinnock et al. [25]). StaRI is targeted on the reporting of interventional implementation studies (the dark shaded box) but will have resonance for studies in the pilot and sustainability phases 
i6795.DC1/pinh034338.w2.pdf. Developed following an e-Delphi and an international consensus meeting, an underpinning theme of StaRI is the distinction between the implementation strategy ('how the intervention was implemented') and the evidence-based intervention that is being implemented. These dual strands run through the reporting standards with a requirement to consider the context, methods, outcomes and implications of both the implementation strategy and the intervention. The resultant clarity will, we hope, not only help authors of implementation papers, but will also be valuable for clinicians, heath service managers and researchers, planning initiatives to disseminate and implement guidelines and improve the quality of care.

In efficacy trials, the aim is to demonstrate that an intervention works when it is delivered as intended; 'fidelity' is thus prioritised and there will be little or no room for clinical or organisational manoeuvre. In contrast, in implementation studies, fidelity will be required to the core features of the intervention, but adaptation of the implementation strategy will be expected-indeed encouraged-to suit local organisational routines, demographic profiles and clinical context. ${ }^{27}$

Another feature of StaRI is the expectation that authors will detail how they believe the implementation strategy and intervention will work to improve health outcomes. This then leads logically to determining the key components of the implementation strategy and the process, implementation and health outcomes that should be measured. This is a discipline that will not only improve the quality of reporting implementation studies, but could also contribute to the development and evaluation of all healthcare initiatives.

One of the key challenges of using StaRI will be including the substantial descriptions of context, implementation strategy and intervention within the permitted word counts. We expect that authors will look for innovative ways to succinctly provide adequate detail (the Explanation and Elaboration document offers some suggestions ${ }^{26}$ ) and npj PCRM encourages on-line supplements for additional information. Publication of protocols, already the norm for randomised trials, will offer opportunity for publishing details of the implementation strategy and intervention. ${ }^{5,7}$

Implementation studies resonate with primary care readers, but poor quality of reporting has made them difficult to identify, and their value is limited if descriptions are inadequate. By expecting adherence to the StaRI standards, npj PCRM hopes to contribute to efforts to improve the standard of reporting of implementation science thereby enhancing its value to researchers and clinicians, and ultimately raising the quality of care for people with respiratory conditions.

\section{AUTHOR CONTRIBUTIONS}

Both authors were co-investigators in the StaRI work. H.P. wrote the first draft which was revised by A.S. Both authors approved the final version.

\section{ADDITIONAL INFORMATION}

Competing interests: The authors are co-investigators in the StaRI work. A.S. is Editor-In-Chief of the npjPCRM.

Publisher's note: Springer Nature remains neutral with regard to jurisdictional claims in published maps and institutional affiliations.

Hilary Pinnock' and Aziz Sheikh'
${ }^{1}$ Asthma UK Centre for Applied Research, Usher Institute of
Population Health Sciences and Informatics, The University of
Edinburgh, Edinburgh EH* 9AG, UK
Correspondence: Hilary Pinnock (hilary.pinnock@ed.ac.uk)

\section{REFERENCES}

1. Honkoop, P. J. et al. Adaptation of IPCRG's difficult to manage asthma programme for implementation in the Dutch context: a modified e-Delphi. npj Prim. Care Respir. Med. 27, 6086 (2017).

2. Martins, S. M. et al. Implementation of 'matrix support' (collaborative care) to improve diagnosis and treatment of asthma and COPD in primary care in Brazil: pilot observational study. npj Prim. Care Respir. Med. 26, 16047 (2016).

3. Panaitescu, C. et al. Barriers to the provision of smoking cessation assistance: a qualitative study among Romanian family physicians. npj Prim. Care Respir. Med. 24, 14022 (2014).

4. Román-Rodríguez, M., Garcia Pardo, M., Gorreto López, L., Uréndez Ruiz, A. \& van Boven, J. F. M. Enhancing the use of asthma and COPD assessment tools in balearic primary care (ACATIB): a region-wide cluster-controlled implementation trial. npj Prim. Care Respir. Med. 26, 16003 (2016).

5. Cragg, L., Williams, S. \& Chavannes, N. H. FRESH AIR: an implementation research project funded through Horizon 2020 exploring the prevention, diagnosis and treatment of chronic respiratory diseases in low-resource settings. npj Prim. Care Respir. Med. 26, 16035 (2016).

6. Hull, S. A. et al. Asthma prescribing, ethnicity and risk of hospital admission: an analysis of 35,864 linked primary and secondary care records in East London. npj Prim. Care Respir. Med. 26, 16049 (2016).

7. Vargas, C. et al. Protocol for regional implementation of collaborative lung function testing. npj Prim. Care Respir. Med. 26, 16024 (2016).

8. Vervloet, M. et al. Reducing antibiotic prescriptions for respiratory tract infections in family practice: results of a cluster randomized controlled trial evaluating a multifaceted peer-group-based intervention. npj Prim. Care Respir. Med. 26, 15083 (2016).

9. Epton, M. J. et al. Development and outcomes of a primary care-based sleep assessment service in Canterbury, New Zealand. npj Prim. Care Respir. Med. 27, 26 (2017).

10. Djandji, F. et al. Enablers and determinants of the provision of written action plans to patients with asthma: a stratified survey of Canadian physicians. npj Prim. Care Respir. Med. 27, 21 (2017).

11. Morrow, S. et al. on behalf of the IMP ${ }^{2}$ ART team. Exploring the perspectives of clinical professionals and support staff on implementing supported selfmanagement for asthma in UK general practice: an IMP ${ }^{2}$ ART qualitative study. npj Prim Care Respir Med. in press (2017).

12. Pinnock, $H$. et al. The international primary care respiratory group (IPCRG) research needs statement 2010. npj Prim. Care Resp. J. 19, S1-S21 (2010).

13. Pinnock, $H$. et al. Prioritising the respiratory research needs of primary care: the international primary care respiratory group (IPCRG) e-Delphi exercise. npj Prim. Care Respir. J. 21, 19-27 (2012).

14. Primary Care Respiratory Society About the PCRS-UK https://pcrs-uk.org/primarycare-respiratory-society-uk (2017).

15. Peters, D. H., Adam, T., Alonge, O., Akua Agyepong, I. \& Tran, N. Implementation research: what it is and how to do it. BMJ 347, f6753 (2013).

16. Foy, R., Sales, A. \& Wensing, M. et al. Implementation science: a reappraisal of our journal mission and scope. Implement. Sci. 10, 51 (2015).

17. Craig, P. et al. Developing and evaluating complex interventions: the new Medical Research Council guidance. London: MRC; 2008. www.mrc.ac.uk/ complexinterventionsguidance (2017).

18. Pinnock, H., Epiphaniou, E. \& Taylor, S. J. C. Phase IV implementation studies: the forgotten finale to the complex intervention methodology framework. Ann. Am. Thorac. Soc. 11, S118-S122 (2014).

19. Lansdverk, J. et al. Design and analysis in dissemination and implementation research. In Dissemination and implementation research in health: translating science into practice (eds Brownson, R. C., Colditz, G. A., Proctor, E. K.) Ch. 12 (Oxford University Press, 2012).

20. Pinnock, H. et al. for the PRISMS group. Systematic meta-review of supported selfmanagement for asthma: a healthcare service perspective. BMC Med. 15, 64 (2017).

21. Pinnock, $H$. et al. for the PRISMS group. Implementing supported selfmanagement for asthma: a systematic review of implementation studies. BMC Med. 13, 127 (2015).

22. Zwarenstein, M. et al. for the CONSORT and Pragmatic Trials in Healthcare (Practihc) groups. Improving the reporting of pragmatic trials: an extension of the CONSORT statement. BMJ 337, a2390 (2008).

23. von Elm, E. et al. for the STROBE initiative. The strengthening the reporting of observational studies in epidemiology (STROBE) statement: guidelines for reporting observational studies. J. Clin. Epidemiol. 61, 344-349 (2008).

24. Tong, A., Sainsbury, P. \& Craig, J. Consolidated criteria for reporting qualitative research (COREQ): a 32-item checklist for interviews and focus groups. Int. J. Qual. Health Care 19, 349-357 (2007). 
25. Pinnock, H. et al. for the StaRl group. Standards for reporting implementation studies (StaRI) statement. BMJ 347, f6753 (2017)

26. Pinnock, $H$. et al. for the StaRi group. Standards for reporting implementation studies (StaRl). Explanation and elaboration document. BMJ Open 7, e013318 (2017)

27. Hawe, P., Shiell, A. \& Riley, T. Complex interventions: how "out of control" can a randomised controlled trial be? BMJ 328, 1561-1563 (2004).

(i) Open Access This article is licensed under a Creative Commons Attribution 4.0 International License, which permits use, sharing, adaptation, distribution and reproduction in any medium or format, as long as you give appropriate credit to the original author(s) and the source, provide a link to the Creative Commons license, and indicate if changes were made. The images or other third party material in this article are included in the article's Creative Commons license, unless indicated otherwise in a credit line to the material. If material is not included in the article's Creative Commons license and your intended use is not permitted by statutory regulation or exceeds the permitted use, you will need to obtain permission directly from the copyright holder. To view a copy of this license, visit http://creativecommons. org/licenses/by/4.0/.

(c) The Author(s) 2017 